\title{
Investigating Pain-Related Avoidance Behavior using a Robotic Arm-Reaching Paradigm
}

Citation for published version (APA):

Glogan, E., Gatzounis, R., Vandael, K., Franssen, M., Vlaeyen, J. W. S., \& Meulders, A. (2020).

Investigating Pain-Related Avoidance Behavior using a Robotic Arm-Reaching Paradigm. Journal of visualized experiment, (164), [e61717]. https://doi.org/10.3791/61717

Document status and date:

Published: 03/10/2020

DOI:

$10.3791 / 61717$

Document Version:

Publisher's PDF, also known as Version of record

Document license:

Taverne

\section{Please check the document version of this publication:}

- A submitted manuscript is the version of the article upon submission and before peer-review. There can be important differences between the submitted version and the official published version of record.

People interested in the research are advised to contact the author for the final version of the publication, or visit the DOI to the publisher's website.

- The final author version and the galley proof are versions of the publication after peer review.

- The final published version features the final layout of the paper including the volume, issue and page numbers.

Link to publication

\footnotetext{
General rights rights.

- You may freely distribute the URL identifying the publication in the public portal. please follow below link for the End User Agreement:

www.umlib.nl/taverne-license

Take down policy

If you believe that this document breaches copyright please contact us at:

repository@maastrichtuniversity.nl

providing details and we will investigate your claim.
}

Copyright and moral rights for the publications made accessible in the public portal are retained by the authors and/or other copyright owners and it is a condition of accessing publications that users recognise and abide by the legal requirements associated with these

- Users may download and print one copy of any publication from the public portal for the purpose of private study or research.

- You may not further distribute the material or use it for any profit-making activity or commercial gain

If the publication is distributed under the terms of Article $25 \mathrm{fa}$ of the Dutch Copyright Act, indicated by the "Taverne" license above, 


\title{
Investigating Pain-Related Avoidance Behavior using a Robotic Arm-Reaching Paradigm
}

\author{
Eveliina Glogan ${ }^{1,2}$, Rena Gatzounis ${ }^{1}$, Kristof Vandael ${ }^{1,3}$, Mathijs Franssen ${ }^{2}$, Johan W. S. Vlaeyen ${ }^{1,2}$, Ann Meulders ${ }^{1,2}$ \\ ${ }^{1}$ Experimental Health Psychology, Maastricht University ${ }^{2}$ Research Group Health Psychology, KU Leuven ${ }^{3}$ Laboratory of Biological Psychology, KU \\ Leuven
}

\section{Corresponding Author}

Ann Meulders

ann.meulders@kuleuven.be

\section{Citation}

Glogan, E., Gatzounis, R., Vandael, K., Franssen, M., Vlaeyen, J.W.S.,

Meulders, A. Investigating Pain-Related Avoidance Behavior using a Robotic Arm-Reaching Paradigm. J. Vis. Exp. (), e61717, doi:10.3791/61717 (2020).

\section{Date Published}

October 2, 2020

\section{DOI}

$10.3791 / 61717$

URL

jove.com/t/61717

\section{Abstract}

Avoidance behavior is a key contributor to the transition from acute pain to chronic pain disability. Yet, there has been a lack of ecologically valid paradigms to experimentally investigate pain-related avoidance. To fill this gap, we developed a paradigm (the robotic arm-reaching paradigm) to investigate the mechanisms underlying the development of pain-related avoidance behavior. Existing avoidance paradigms (mostly in the context of anxiety research) have often operationalized avoidance as an experimenter-instructed, low-cost response, superimposed on stimuli associated with threat during a Pavlovian fear conditioning procedure. In contrast, the current method offers increased ecological validity in terms of instrumental learning (acquisition) of avoidance, and by adding a cost to the avoidance response. In the paradigm, participants perform arm-reaching movements from a starting point to a target using a robotic arm, and freely choose between three different movement trajectories to do so. The movement trajectories differ in probability of being paired with a painful electrocutaneous stimulus, and in required effort in terms of deviation and resistance. Specifically, the painful stimulus can be (partly) avoided at the cost of performing movements requiring increased effort. Avoidance behavior is operationalized as the maximal deviation from the shortest trajectory on each trial. In addition to explaining how the new paradigm can help understand the acquisition of avoidance, we describe adaptations of the robotic arm-reaching paradigm for (1) examining the spread of avoidance to other stimuli (generalization), (2) modeling clinical treatment in the lab (extinction of avoidance using response prevention), as well as (3) modeling relapse, and return of avoidance following extinction (spontaneous recovery). Given the increased ecological validity, and numerous possibilities for extensions and/or adaptations, the robotic arm-reaching paradigm offers a promising tool to facilitate the 


\section{Introduction}

Avoidance is an adaptive response to pain signaling bodily threat. Yet, when pain becomes chronic, pain and painrelated avoidance lose their adaptive purpose. In line with this, the fear-avoidance model of chronic pain ${ }^{1,2,3,4,5,6,7,8}$ posits that erroneous interpretations of pain as catastrophic, trigger increases in fear of pain, which motivate avoidance behavior. Excessive avoidance can lead to the development and maintenance of chronic pain disability, due to physical disuse and decreased engagement in daily activities and aspirations $1,2,3,4,5,9$. Furthermore, given that the absence of pain can be misattributed to avoidance rather than recovery, a self-sustaining cycle of pain-related fear and avoidance can be established $^{10}$.

Despite recent interest in avoidance in the anxiety literature ${ }^{11,12}$, research on avoidance in the pain domain is still in its infancy. Previous anxiety research, guided by the influential two-factor theory ${ }^{13}$, has generally assumed fear to drive avoidance. Correspondingly, traditional avoidance paradigms ${ }^{12}$ entail two experimental phases, each corresponding to one factor: the first to establish fear (Pavlovian conditioning ${ }^{14}$ phase), and the second to examine avoidance (Instrumental ${ }^{15}$ phase). During differential Pavlovian conditioning, a neutral stimulus (conditioned stimulus, CS+; e.g., a circle) is paired with an intrinsically aversive stimulus (unconditioned stimulus, US; e.g., an electric shock), which naturally produces unconditioned responses (URs, e.g., fear). A second control stimulus is never paired with the US (CS-; e.g., a triangle). Following pairings of the CSs with the US, the CS+ will elicit fear in itself (conditioned responses, CRs) in the absence of the US. The CS- comes to signal safety and will not trigger CRs. Afterwards, during instrumental conditioning, participants learn that their own actions (responses, R; e.g., button-press) lead to certain consequences (outcomes; $\mathrm{O}$, e.g., the omission of shock $)^{15,16}$. If the response prevents a negative outcome, the chance of that response recurring increases; this is referred to as negative reinforcement ${ }^{15}$. Thus, in the Pavlovian phase of traditional avoidance paradigms, participants first learn the CS-US association. Subsequently, in the instrumental phase, an experimenterinstructed avoidance response $(R)$ is introduced, canceling the US if performed during CS presentation, establishing a R-O association. Thus, the CS becomes a discriminative stimulus $\left(S^{D}\right)$, indicating the appropriate moment for, and motivating the performance of, the conditioned $\mathrm{R}^{15}$. Apart from some experiments showing instrumental conditioning of pain reports ${ }^{17}$ and pain-related facial expressions ${ }^{18}$, investigations into the instrumental learning mechanisms of pain, in general, are limited.

Although the standard avoidance paradigm, described above, has elucidated many of the processes underlying avoidance, it also has several limitations ${ }^{5,19}$. First, it does not allow examining the learning, or acquisition, of avoidance itself, because the experimenter instructs the avoidance response. Having participants freely choose between multiple trajectories, and, therefore, learn which responses are painful/safe and which trajectories to avoid/ not avoid, more accurately models real-life, where avoidance 
emerges as a natural response to pain ${ }^{9}$. Second, in traditional avoidance paradigms, the button-press avoidance response comes at no cost. However, in real life, avoidance can become extremely costly for the individual. Indeed, high-cost avoidance especially disrupts daily functioning ${ }^{5}$. For example, avoidance in chronic pain can severely limit people's social and working lives ${ }^{9}$. Third, dichotomous responses such as pressing/not pressing a button also do not very well represent real life, where different degrees of avoidance occur. In the following sections, we describe how the robotic arm-reaching paradigm ${ }^{20}$ addresses these limitations, and how the basic paradigm can be extended to multiple novel research questions.

\section{Acquisition of avoidance}

In the paradigm, participants use a robotic arm to perform arm-reaching movements from a starting point to a target. Movements are employed as the instrumental response because they closely resemble pain-specific, fear-evoking stimuli. A ball virtually represents participants' movements onscreen (Figure 1), allowing participants to follow their own movements in real-time. During each trial, participants freely choose between three movement trajectories, represented on-screen by three arches (T1-T3), differing from each other in terms of how effortful they are, and in the likelihood that they are paired with a painful electrocutaneous stimulus (i.e., pain stimulus). Effort is manipulated as deviation from the shortest possible trajectory and increased resistance from the robotic arm. Specifically, the robot is programmed such that resistance increases linearly with deviation, meaning that the more participants deviate, the more force they need to exert on the robot. Furthermore, pain administration is programmed such that the shortest, easiest trajectory (T1) is always paired with the pain stimulus (100\% pain/no deviation or resistance). A middle trajectory (T2) is paired with a $50 \%$ chance of receiving the pain stimulus, but more effort is required (moderate deviation and resistance). The longest, most effortful trajectory (T3) is never paired with the pain stimulus but requires the most effort to reach the target ( $0 \%$ pain/largest deviation, strongest resistance). Avoidance behavior is operationalized as the maximum deviation from the shortest trajectory (T1) per trial, which is a more continuous measure of avoidance, than for example, pressing or not pressing a button. Furthermore, the avoidance response comes at the cost of increased effort. Moreover, given that participants freely choose between the movement trajectories, and are not explicitly informed about the experimental R-O (movement trajectory-pain) contingencies, avoidance behavior is instrumentally acquired. Online selfreported fear of movement-related pain and pain-expectancy have been collected as measures of conditioned fear toward the different movement trajectories. Pain-expectancy is also an index of contingency awareness and threat appraisal ${ }^{21}$. This combination of variables allows scrutinizing the interplay between fear, threat appraisals, and avoidance behavior. Using this paradigm, we have consistently demonstrated the experimental acquisition of avoidance $20,22,23,24$.

\section{Generalization of avoidance}

We have extended the paradigm to investigate generalization of avoidance ${ }^{23}$ - a possible mechanism leading to excessive avoidance. Pavlovian fear generalization refers to the spreading of fear to stimuli or situations (generalization stimuli, GSs) resembling the original CS+, with fear declining with decreasing similarity to the CS+ (generalization gradient) $25,26,27,28$. Fear generalization minimizes the need to learn relationships between stimuli anew, allowing swift detection of novel threats in everchanging environments $25,26,27,28$. However, excessive generalization leads to fear of safe stimuli (GSs similar to 
CS-), thus causing unnecessary distress ${ }^{28}, 29$. In line with this, studies using Pavlovian fear generalization consistently show that chronic pain patients excessively generalize pain-related fear $30,31,32,33,34$, whereas healthy controls show selective fear generalization. Yet, where excessive fear causes discomfort, excessive avoidance can culminate in functional disability, due to avoidance of safe movements and activities, and increased daily activity disengagement ${ }^{1,2,3,4,9}$. Despite its key role in chronic pain disability, research on the generalization of avoidance is scarce. In the paradigm adapted for studying generalization of avoidance, participants first acquire avoidance, following the procedure described above $e^{20}$. In a subsequent generalization phase, three novel movement trajectories are introduced in the absence of the pain stimulus. These generalization trajectories (G1-G3) lie on the same continuum as the acquisition trajectories, resembling each of these trajectories, respectively. Specifically, generalization trajectory $\mathrm{G} 1$ is situated between T1 and T2, G2 between T2 and T3, and G3 to the right of T3. In this way, generalization of avoidance to novel safe trajectories can be examined. In a previous study, we showed generalization of selfreports, but not avoidance, possibly suggesting different underlying processes for pain-related fear- and avoidance generalization $^{23}$.

\section{Extinction of avoidance with response prevention}

The primary method of treating high fear of movement in chronic musculoskeletal pain is exposure therapy ${ }^{35}-$ the clinical counterpart to Pavlovian extinction ${ }^{36}$, i.e., the reduction of CRs through repeated experience with the $\mathrm{CS}+$ in the absence of the $\mathrm{US}^{36}$. During exposure for chronic pain, patients perform feared activities or movements in order to disconfirm catastrophic beliefs and expectations of harm 34,37 . Since these beliefs do not necessarily concern pain per se, but rather underlying pathology, movements are not always carried out pain-free in the clinic ${ }^{34}$. According to inhibitory learning theory ${ }^{38,39}$, extinction learning does not erase the original fear memory (e.g., movement trajectory-pain); rather, it creates a novel inhibitory extinction memory (e.g., movement trajectoryno pain), which competes with the original fear memory for retrieval 40,41 . The novel inhibitory memory is more context-dependent than the original fear memory ${ }^{40}$, deeming the extinguished fear memory susceptible to re-emergence (return of fear) $40,41,42$. Patients are often prevented from performing even subtle avoidance behaviors during exposure treatment (extinction with response prevention, RPE), to establish genuine fear extinction by preventing the misattribution of safety to avoidance 10,43 .

\section{Return of avoidance}

Relapse in terms of return of avoidance is still common in clinical populations, even after extinction of fear $43,44,45,46$. Although multiple mechanisms have been found to result in the return of fear ${ }^{47}$, little is known about those relating to avoidance ${ }^{22}$. In this manuscript, we specifically describe spontaneous recovery, i.e., return of fear and avoidance due to the passage of time 40,47 . The robotic arm-reaching paradigm has been implemented in a 2-day protocol to investigate return of avoidance. During day 1, participants first receive acquisition training in the paradigm, as described above $^{20}$. In a subsequent RPE phase, participants are prevented from performing the avoidance response, i.e., they can only perform the pain-associated trajectory (T1) under extinction. During day 2, to test for spontaneous recovery, all trajectories are available again, but in the absence of pain stimuli. Using this paradigm, we showed that, one day after successful extinction, avoidance returned ${ }^{22}$. 


\section{Protocol}

The protocols presented here meet the requirements of the Social and Societal Ethics committee of the KU Leuven (registration number: S-56505), and the Ethics Review Committee Psychology and Neuroscience of Maastricht University (registration numbers: 185_09_11_2017_S1 and 185_09_11_2017_S2_A1).

\section{Preparing the laboratory for a test session}

1. Before the test session: Send the participant an e-mail informing him/her about the delivery of pain stimuli, of the general outline of the experiment, and the exclusion criteria. Exclusion criteria for healthy participants comprise: being under 18 years of age; chronic pain; analphabetism or diagnosed dyslexia; pregnancy; lefthandedness; current/history of cardiovascular disease, chronic or acute respiratory disease (e.g., asthma, bronchitis), neurological disease (e.g., epilepsy), and/ or psychiatric disorder (e.g., clinical depression, panic/ anxiety disorder); uncorrected problems with hearing or vision; having pain in the dominant hand, wrist, elbow or shoulder that may hinder performing the reaching task; presence of implanted electronic medical devices (e.g., cardiac pacemaker); and presence of any other severe medical conditions.

2. Due to COVID-19 safety precautions, ask the participant to wash/disinfect his/her hands upon arrival at the lab, and do so yourself. Wear a disposable facemask throughout the duration of the test session, and latex gloves whenever physical contact with the participant is required.
3. Use two separate rooms or sections for the experimental setting: one for the participant and the other for the experimenter.

4. Use one computer with two separate screens: one computer screen for the experimenter, and one larger television screen for the participant.

5. To turn on the robotic arm (e.g., HapticMaster), press the power switch in the front of the robot (specific to this robot). Subsequently, turn on the emergency switch, which may later be used to turn off the robot if necessary.

6. Recalibrate the robotic arm before each test day. This is done via a direct application programming interface (API) connection with the robotic arm, and only needs to be done once, at the beginning of the test day.

1. To establish the API connection, open an internet browser on the computer, and type in the specific API address of the robotic arm.

2. On the webpage, select State under HapticMASTER. Subsequently, press the Start button next to Init (for initialize).

NOTE: This is the standard calibration procedure for this robot. Different robots may require different calibration procedures.

7. Use a constant current stimulator, which is connected to the computer (see step 1.4). During the experiment, the pain stimulus is delivered via the experimental script, which runs on the computer. The experiment is programmed using a cross-platform game engine (see Table of Materials).

1. For safety reasons, disable the constant current stimulator output by switching down the orange toggle 
switch in the upper-right corner of the stimulator's front control panel.

2. Use the orange toggle switch in the middle of the front control panel to set the output range to $x 10 \mathrm{~mA}$.

3. Use the black rotary knob in the upper-left corner of the front control panel to set the pulse duration to 2 ms $(2000 \mu s)$.

4. To switch on the constant current stimulator, press the power button in the lower-left corner of the front control panel.

\section{Screening for exclusion criteria and obtaining informed consent}

1. Position the participant approximately $2.5 \mathrm{~m}$ from the television screen (see step 1.4), at a comfortable distance $(\sim 15 \mathrm{~cm})$ from the handle (sensor) of the robotic arm, in a chair with arm rests (Figure 1).

2. Screen the participant for exclusion criteria by means of self-report (see step 1.1 for exclusion criteria).

3. Inform the participant about the delivery of pain stimuli and of the general outline of the experiment. Also, inform $\mathrm{him} / \mathrm{her}$ that he/she is free to withdraw participation at any point during the experiment, without any repercussions. Obtain written informed consent.

4. To minimize physical contact with the participant, ensure that the participant section of the lab includes a table on which exclusion and informed consent forms, as well as a Tablet for questionnaires (see step 6.2) are placed before the participant's arrival. The participant should be able to access and sign the forms independently using this table.

\section{Attaching the stimulation electrodes}

NOTE: The pain stimulus is a $2 \mathrm{~ms}$ square-wave electrical stimulus delivered cutaneously through two stainless steel bar stimulation electrodes (electrode diameter $8 \mathrm{~mm}$, interelectrode distance $30 \mathrm{~mm}$ ).

1. If the participant is wearing long sleeves, ask him/her to roll up the sleeve on his/her right arm at least $10 \mathrm{~cm}$ above his/her elbow.

2. Fill the center of the stimulation electrodes with conductive electrolyte gel and plug the electrode cables to the emergency switch, which is connected to the constant current stimulator in the experimenter section of the lab.

3. Attach the stimulation electrodes over the triceps tendon of the participant's right arm using a strap. Make sure the strap is neither too tight nor too loose. Once the electrodes have been attached, tell the participant to relax his/her arm

\section{Calibrating the pain stimulus}

1. Explain the pain calibration procedure and corresponding scale by presenting it on the television screen (see step 1.4).

1. Clarify to the participant that he/she may choose the stimulus which he/she will receive during the experiment, but explain that for data integrity he/she is asked to select a stimulus that he/she would describe as "significantly painful and demanding some effort to tolerate".

2. Ask the participant to rate each stimulus on the numerical scale presented on the television screen, ranging from $0-10$, where 0 is labeled as "I feel nothing"; 1 as "I feel something, but this is not 
unpleasant; it is only a sensation" (i.e., detection threshold), 2 as "the stimulus is not yet painful, but is beginning to be unpleasant"; 3 as "the stimulus starts being painful" (i.e. pain threshold); and 10 as "this is the worst pain I can imagine".

2. Enable the constant current stimulator output by switching up the orange toggle switch (see step 1.7.1).

3. During the pain calibration procedure, manually increase the intensity of the pain stimuli using the rotary knob on the front control panel of the constant current stimulator. The intensity of the pain stimulus can be seen above this knob.

1. Start with an intensity of $1 \mathrm{~mA}$, and gradually increase the intensity in a stepwise manner, with increases of $1,2,3$, and $4 \mathrm{~mA}$ increments. Use the following order of stimulus presentations in mA: 1, 2, 4, 6, 8, 11, 14, $17,20,24,28,32,36,40,44,48,52$, etc.

4. To deliver the pain stimuli one stimulus at a time, manually trigger the constant current stimulator by pressing the orange trigger button on the front control panel.

1. Announce each stimulus to the participant before triggering the constant current stimulator.

5. Terminate the calibration procedure once the participant reaches a pain intensity level which he/she would describe as "significantly painful and demanding some effort to tolerate". Ideally, this should correspond to a 78 on the pain calibration rating scale.

6. Document the participant's final pain intensity in $\mathrm{mA}$ and his/her pain intensity rating $(0-10)$ and maintain this intensity for the remainder of the experiment.

\section{Running the experimental task}

1. Verbally inform the participant that he/she will receive instructions about the robotic arm-reaching paradigm on the television screen in front of him/her, after which he/ she will be able to practice the task under the supervision of the experimenter.

2. Provide the participant with standardized written instructions of the task on-screen.

3. Practice: Via the experimental script, on the television screen, present three arches (T1-T3) situated midway through the movement plane. The easiest arm movement (T1) is paired with no deviation or resistance, the middle arm movement (T2) is paired with moderate deviation and resistance, and the furthest arm movement (T3) is paired with the largest deviation and strongest resistance.

1. Instruct the participant to use his/her dominant hand to operate the sensor of the robotic arm, represented by a green ball on the television screen, and to move the ball/sensor from a starting point at the lowerleft corner of the movement plane, to a target at the upper-left corner of the movement plane.

2. Instruct the participant that he/she can freely choose which one of the available movement trajectories to perform on each trial.

4. Do not administer the pain stimulus (see section 3: Note and step 5.7.6) during the practice phase. However, ensure that the relationship between deviation and resistance (see step 5.3) is in place.

5. Provide the participant with verbal feedback while they perform the practice phase.

1. Ensure the participant does not start moving before the visual and auditory "start signals", and that he/she 
releases the robotic arm immediately when the visual and auditory "stop signals" are presented.

NOTE: Two distinct auditory signals (a "start tone" and a "scoring tone") and two distinct visual signals (the target and a virtual "traffic light" turning green and red, respectively; Figure 1) have been used as start and stop signals. Auditory and visual start signals are presented simultaneously, as are auditory and visual stop signals.

2. Instruct the participant to provide self-report measures of pain-expectancy and fear of movementrelated pain on a continuous rating scale, by scrolling to the left and right on the scale using two respective foot pedals on a triple foot switch. Instruct him/her to confirm his/her answer using a third foot pedal.

NOTE: Present self-report questions on fixed, predetermined trials, for each movement trajectory separately. Ensure, via the experimental script, that the robotic arm is immobilized and remains fixed during the time the participant is responding to the questions.

6. At the end of the practice phase, respond to the participant's questions. Leave the experimental section/ room and dim the lights. The participant starts the experiment himself/herself by pressing the 'Confirm' foot pedal (see step 5.5.2).

7. Acquisition: During avoidance acquisition, similarly to the practice phase, let the participant choose which movement trajectory (T1-T3) to perform on each trial.

1. During avoidance acquisition, subject the participant to the experimental Response-Outcome (movement trajectory-pain) contingencies, and to the avoidance- costs, i.e., the tradeoff between pain and effort, via the experimental script.

2. Specifically, if the participant performs the easiest movement trajectory (T1), always present the pain stimulus (100\% pain/no deviation or resistance).

3. If he/she performs the middle movement trajectory (T2), present the pain stimulus with a $50 \%$ chance, but ensure he/she will have to exert more effort (moderate deviation and resistance).

4. If the participant performs the furthest, most effortful movement trajectory (T3), do not present the pain stimulus at all, but ensure that he/she will have to exert the most effort to reach the target $(0 \%$ pain/ largest deviation, strongest resistance).

NOTE: If applicable to the design, a Yoked Group can be used as control. In yoked procedures, each control participant is paired with a participant in the experimental group, such that the two receive the same reinforcement schedules ${ }^{48}$. Thus, in the current paradigm, each Yoked Group participant receives pain stimuli on the same trials as his/ her Experimental Group counterpart, regardless of the trajectories he/she chooses. No acquisition of avoidance behavior is expected in the Yoked Group, given the lack of manipulated Response-Outcome (movement trajectory-pain) contingencies.

5. Where applicable, save data from each Experimental Group participant on the computer (see section 1.4), and use as reference for the reinforcement schedules of each Yoked (control) Group participant.

1. If using a Yoked procedure (i.e., each control participant is paired with a participant in the experimental group, such that the two receive 
the same reinforcement schedules ${ }^{48}$ ), allocate participants to groups using a randomization schedule with the rule that the first participant must be in the Experimental Group. Following this, participants are assigned to either group randomly, as long as, at each point, the number of Experimental Group participants exceeds the number of Yoked Group participants.

6. On trials with a pain stimulus, present the pain stimulus once two-thirds of the movement has been performed, i.e., once the participant has moved through a trajectory arch. The constant current stimulator is automatically triggered via the experimental script.

7. Successful trial-completion is indicated by the presentation of visual and auditory stop signals. Subsequently, ensure, via the experimental script, that the robotic arm automatically returns to its starting position where it remains fixed. After $3,000 \mathrm{~ms}$, present the visual and auditory start signals, and the participant can start the next trial.

NOTE: Trial duration differs between trials and participants, due to differences in movement speeds. The number of trials per experimental phase can also change between experiments. We recommend a minimum of $2 \times 12$ trials for successful acquisition of avoidance. Including the steps described above, the acquisition protocol lasts approximately $45 \mathrm{~min}$.

8. Generalization: In the generalization protocol, test for generalization of avoidance after the acquisition phase (see section 5.7).

NOTE: When testing for generalization of avoidance, the on-screen trajectory arches are separated during acquisition, to leave room for the generalization trajectory arches, which are positioned between the acquisition trajectory arches (see Figure 1).

1. On the television screen, present three novel movement trajectories instead of the acquisition trajectories T1-T3. Ensure that these "generalization trajectories" (G1-G3) are located adjacent to the acquisition trajectories. Specifically, G1 is situated between T1 and T2, G2 between T2 and T3, and G3 to the right of T3 (see Figure 1). Do not pair generalization trajectories with the pain stimulus.

NOTE: Including the steps described above, with a generalization phase of $3 \times 12$ trials, the avoidance generalization protocol lasts approximately $1.5 \mathrm{~h}$. A Yoked Group ${ }^{48}$ is required for testing generalization of avoidance (see step 5.7.5). However, different controls can be used depending on the specific research question (cf. context modulation of avoidance in a within-subjects design ${ }^{24}$ ).

9. Extinction with response prevention (RPE): In the RPE protocol, after the acquisition phase (see section 5.7), provide the participants with standardized written instructions stating that in the upcoming phase they are only allowed to perform $\mathrm{T} 1$.

1. During the RPE phase, via the experimental script, visually (e.g., blocking the trajectory arches with a gate) and/or haptically (e.g., block participant's arm movement with a haptic wall) block T2 and T3, so that only $\mathrm{T} 1$ is available. T1 is not paired with the pain stimulus during this phase. Including the steps described above, with an RPE phase of $4 \times 12$ trials, this session lasts approximately $60 \mathrm{~min}$.

10. Test of spontaneous recovery: For testing spontaneous recovery of avoidance, administer a 2-day protocol with 
$24 \mathrm{~h} \pm 3 \mathrm{~h}$ in between sessions. On day 1 , administer the RPE protocol (see section 5.9).

1. On day 2, attach the stimulation electrodes (see section 3). Provide brief on-screen refresher instructions of the task. Do not include any information regarding the pain stimuli.

2. Present the three acquisition trajectories (T1-T3, cf. acquisition phase, see section 5.7), in the absence of the pain stimulus. Including the post-experimental questionnaire (see section 6.2), and a spontaneous recovery phase of $4 \times 12$ trials, this session lasts approximately $45 \mathrm{~min}$.

NOTE: To prevent reinstatement of fear (i.e., return of fear following unexpected encounters with the pain stimulus $^{42}$; see discussion), do not recalibrate the pain stimulus on day 2 .

\section{Concluding the experiment}

1. Once the participant has completed the experiment, detach the stimulation electrodes.

2. Provide the participant with a Tablet located on the table in the participant's section of the lab (see section 2.4), for responding to an exit questionnaire inquiring about the intensity and unpleasantness of the pain stimulus and avoidance-costs, as well as awareness of the experimental Response-Outcome (movement trajectorypain) contingencies.

3. While the participant completes the psychological trait questionnaires, clean off the electrolyte gel from the stimulation electrodes.

4. Once the participant has finished completing the psychological trait questionnaires, provide him/her with a debriefing and reimbursement.
5. Clean the stimulation electrodes thoroughly with a disinfectant solution appropriate for cleaning medical instruments; remove all the gel inside and around the electrodes. Dry the electrodes with soft tissue paper. Clean the sensor of the robotic arm with disinfectant wipes or spray.

\section{Representative Results}

Acquisition of avoidance behavior is demonstrated by participants avoiding more (showing larger maximal deviations from the shortest trajectory) at the end of an acquisition phase, compared to the beginning of the acquisition phase (Figure 2, indicated by $A)^{20}$, or as compared to a Yoked control group (Figure 3) ${ }^{23,48}$.

Acquisition of fear and pain-expectancy is evidenced by participants reporting lower fear for T3 compared to T1 and $\mathrm{T} 2$, and expecting the pain stimulus less during T3 compared to $T 1$ and $T 2^{20}$. Differential self-reports between $T 1$ and T3 are of primary interest, because T2 is ambiguous. Nondifferential self-reports between T1 and T2 have also been found, with both differing from $\mathrm{T}^{23}$ (Figure 4A, Figure 5A, Figure 6A, and Figure 7A).

Acquisition is a prerequisite for generalization. Generalization of avoidance behavior is indicated by participants in the Experimental Group avoiding (deviating) more than the Yoked Group ${ }^{48}$ at the beginning of the generalization phase. Given that generalization is tested in the absence of pain stimuli, avoidance behavior may decrease throughout the generalization phase. Furthermore, a general decrease in avoidance behavior between the end of the acquisition phase and the beginning of the generalization phase (generalization decrement) can be expected. This is a result of the introduction of novel movement trajectories, which may 
constitute a context-switch ${ }^{49}, 50$. In a previous study, we did not find generalization of avoidance, possibly due to specific parameters of the paradigm ${ }^{23}$.

Generalization of fear and pain-expectancy is indicated by a similar pattern to that of the acquisition phase, i.e., by participants in the Experimental Group reporting lower fear to $\mathrm{G} 3$ compared to $\mathrm{G} 1$ and $\mathrm{G} 2$, and expecting the pain stimulus less during $G 3$ compared to $G 1$ and $G 2$, at the beginning of the generalization phase. As in the acquisition phase, differential self-reports between G1 and G3 are of primary interest (Figure 4B and Figure 5B). Non-differential self-reports between $\mathrm{G} 1$ and $\mathrm{G} 2$ have been reported so far, with both differing from $\mathrm{G}^{23}$. Furthermore, given that generalization is tested in the absence of pain stimuli, participants may report less fear and pain-expectancies throughout the generalization phase. Furthermore, a general decrease in fear and pain-expectancies toward the novel generalization trajectories, compared to the acquisition trajectories (generalization decrement) can be expected. In a previous study, we found generalization of fear and painexpectancies, despite avoidance not generalizing ${ }^{23}$.

Acquisition is a prerequisite for extinction. During extinction of avoidance behavior with response prevention, participants are only allowed to perform the previously painful movement trajectory (T1), whereas the other two trajectories ( $\mathrm{T} 2$ and T3) are prohibited. Therefore, given that participants only have the option of performing $\mathrm{T} 1$, and thus the observed data pattern does not reflect their own choices, i.e., genuine extinction of avoidance behavior, extinction of avoidance is not included in the analyses (Figure 2).

Extinction of fear and pain-expectancies is evident when participants report lower fear for $\mathrm{T} 1$ and expect the pain stimulus less when performing $\mathrm{T} 1$, at the end of the RPE phase, compared to the end of the acquisition phase. (Figure 6B and Figure 7B).

Extinction of self-report measures is a prerequisite for spontaneous recovery. Spontaneous recovery of avoidance behavior is indicated by participants avoiding more at the beginning of the test of spontaneous recovery, compared to the end of the RPE phase (Figure 2B).

Spontaneous recovery of fear and pain-expectancy is indicated by participants reporting higher fear and painexpectancy for $\mathrm{T} 1$, during the beginning of the test of spontaneous recovery, compared to the end of the RPE phase (Figure 6C and Figure 7C). 


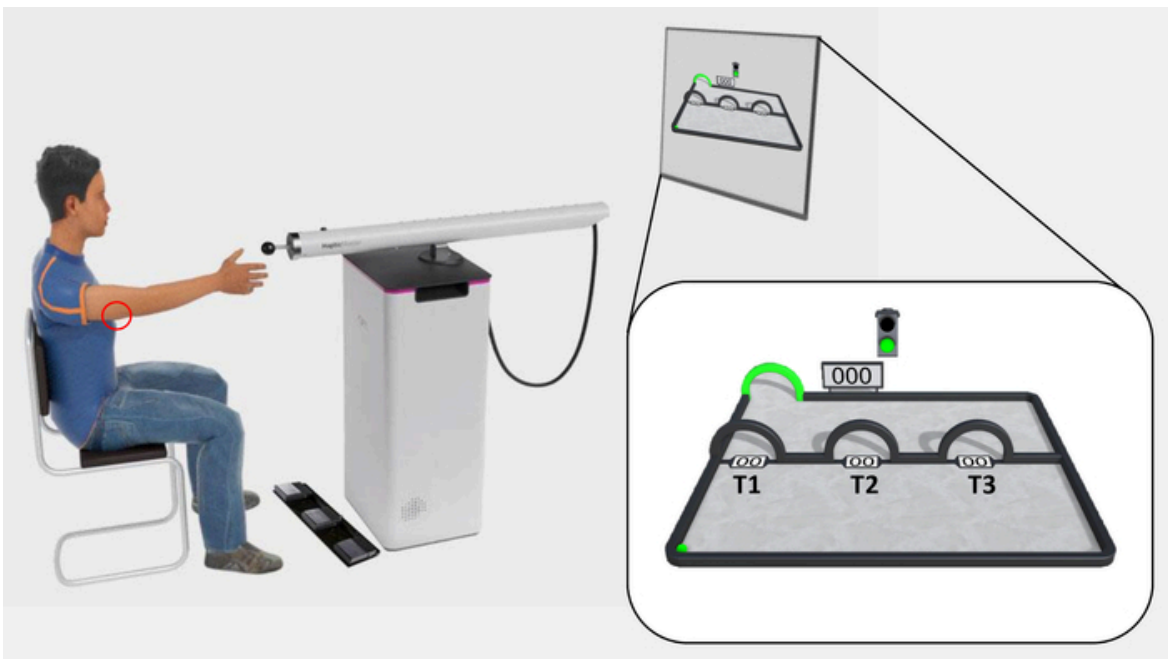

Figure 1: The experimental set-up and outlook of the experimental task. The participant is seated in front of the television screen, at reaching distance from the sensor of the robotic arm. The electrodes are placed on the triceps tendon of the right arm, where the pain stimuli are delivered (red circle), and the triple foot switch is used to give fear of movementrelated pain and pain-expectancy ratings. The acquisition phase of the experimental task is shown on the television screen and magnified in the white box. The ball is situated in the lower-left corner, and the target in the upper-left corner (green arch). T1-T3 are situated midway through the movement-plane, from left to right, respectively. Spaces are left between T1T3 specifically in avoidance generalization protocols, in order to leave room for the subsequent generalization trajectory arches (G1-G3). Please click here to view a larger version of this figure. 


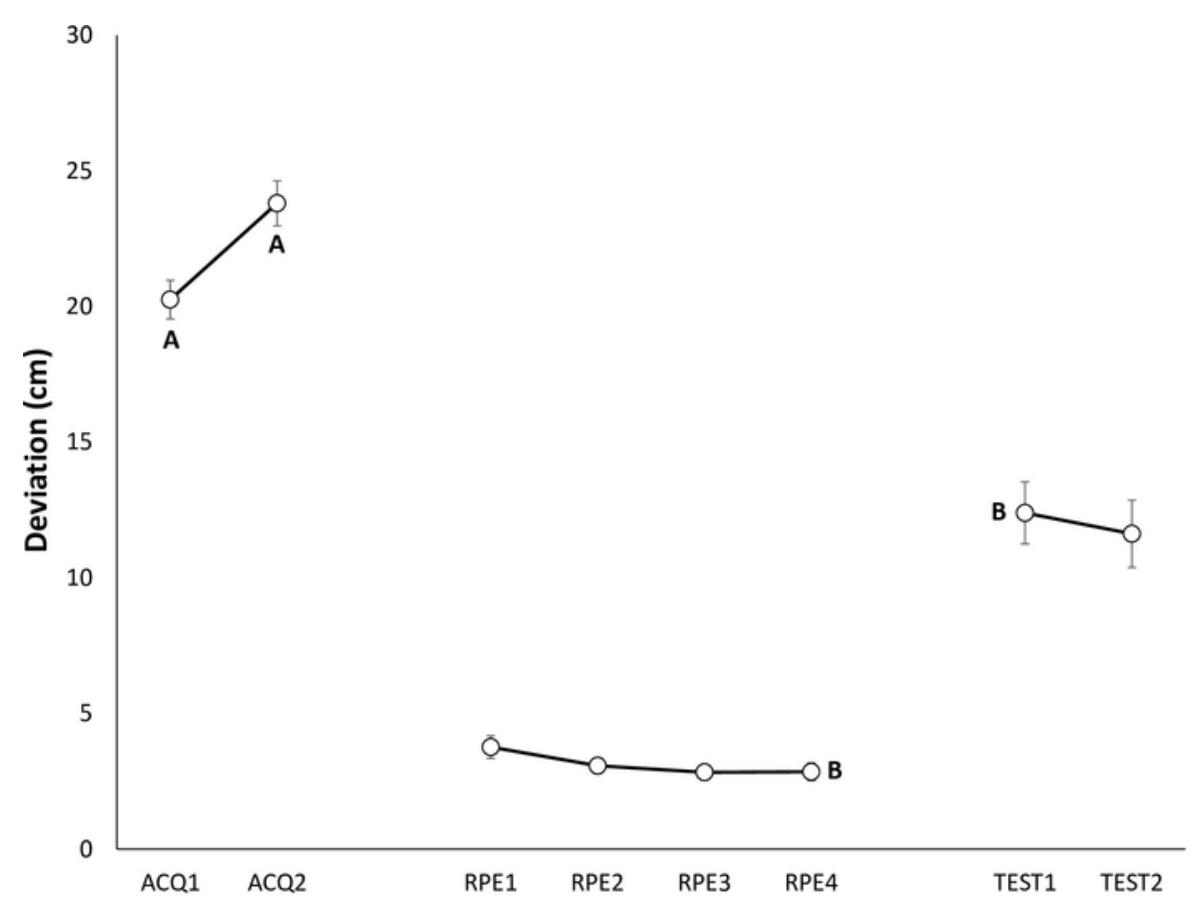

Figure 2: Representative data of avoidance behavior during the acquisition, extinction with response prevention, and test of spontaneous recovery phases ${ }^{22}$. Mean maximum deviation (in centimeters) from the shortest trajectory to the target during acquisition (ACQ1-2), extinction with response prevention (RPE1-4), and spontaneous recovery (TEST1-2). Note that, participants are only allowed to perform the shortest trajectory (T1) during the RPE phase. Error bars represent standard error of the mean (SEM). Data in this figure are from 30 participants $(9 \text { men, } 21 \text { women; mean age }=21.90)^{22}$. This figure is modified with permission from ref. ${ }^{22}$. Please click here to view a larger version of this figure. 


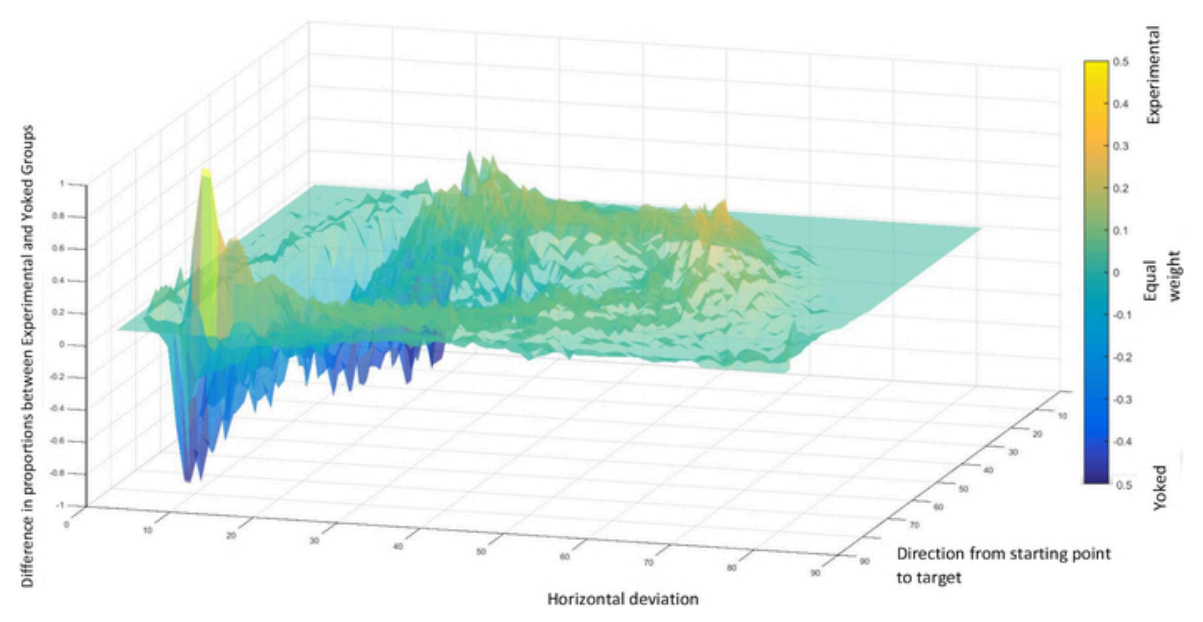

Figure 3: Representative data of avoidance behavior during the acquisition phase ${ }^{20}$. Relative proportions of movements between the Experimental and Yoked ${ }^{48}$ Groups, within the experimental movement plane. Top, yellow patterns represent movements predominantly performed by the Experimental Group, and bottom, blue patterns represent movements predominantly performed by the Yoked Group. "Direction from starting point to target" indicates the shortest possible trajectory from the starting point to the target. "Horizontal deviation" indicates deviation from the shortest possible movement trajectory. Data in this figure are from 50 participants (36 men, 14 women; mean age $=24.92)^{20}$. This figure is reprinted with permission from ref. ${ }^{20}$. Please click here to view a larger version of this figure. 


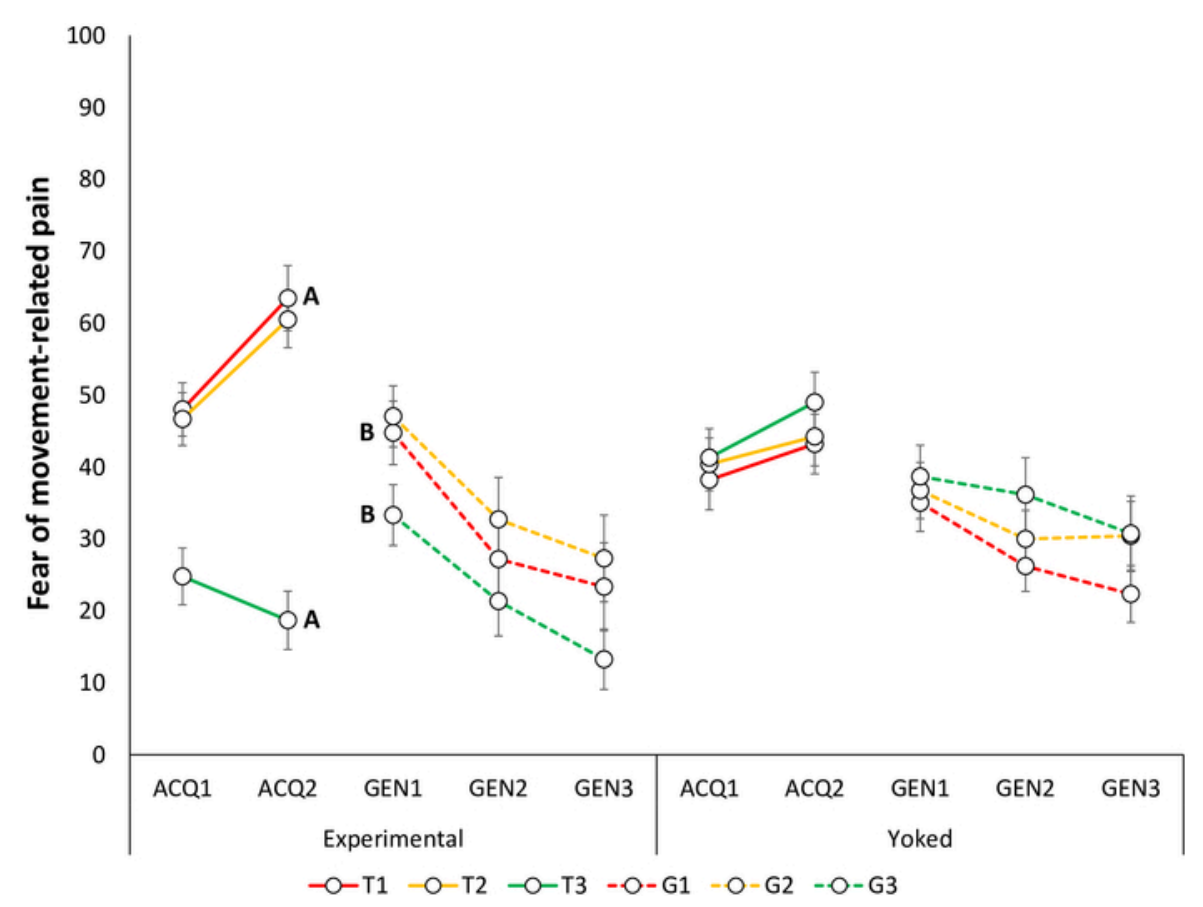

Figure 4: Representative data of fear of movement-related pain during the acquisition and generalization

phases $^{23}$. Mean fear of movement-related pain toward the acquisition trajectories in the Experimental and Yoked ${ }^{48}$ groups $^{2}$ during the acquisition blocks (ACQ1-3), and generalization blocks (GEN1-3). Note that during the acquisition phase, selfreports are provided for trajectories T1-T3 and during the generalization phase, for G1-G3. Error bars represent SEM. Data in this figure are from 64 participants (32 per group; Experimental Group: 10 men, 22 women, mean age = 22.88; Yoked Group: 12 men, 20 women; mean age $=23.44)^{23}$. This figure is modified with permission from ref. ${ }^{23}$. Please click here to view a larger version of this figure. 


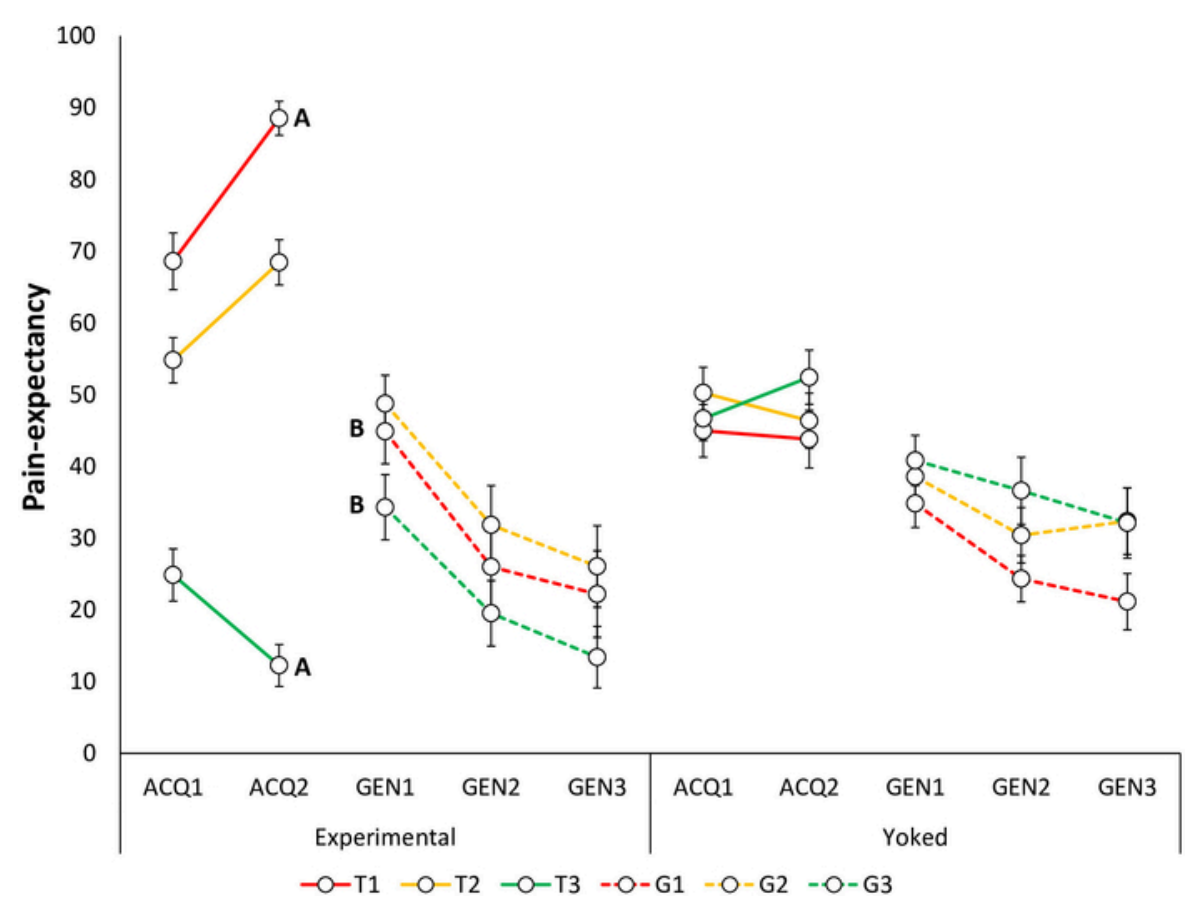

Figure 5: Representative data of pain-expectancy during the acquisition and generalization phases ${ }^{23}$. Mean painexpectancy toward the acquisition trajectories in the Experimental and Yoked ${ }^{48}$ groups during the acquisition blocks (ACQ13), and generalization blocks (GEN1-3). Note that during the acquisition phase, self-reports are provided for trajectories T1T3 and during the generalization phase, for G1-G3. Error bars represent SEM. Data in this figure are from 64 participants (32 per group; Experimental Group: 10 men, 22 women, mean age = 22.88; Yoked Group: 12 men, 20 women; mean age = $23.44)^{23}$. This figure is modified with permission from ref. ${ }^{23}$. Please click here to view a larger version of this figure. 


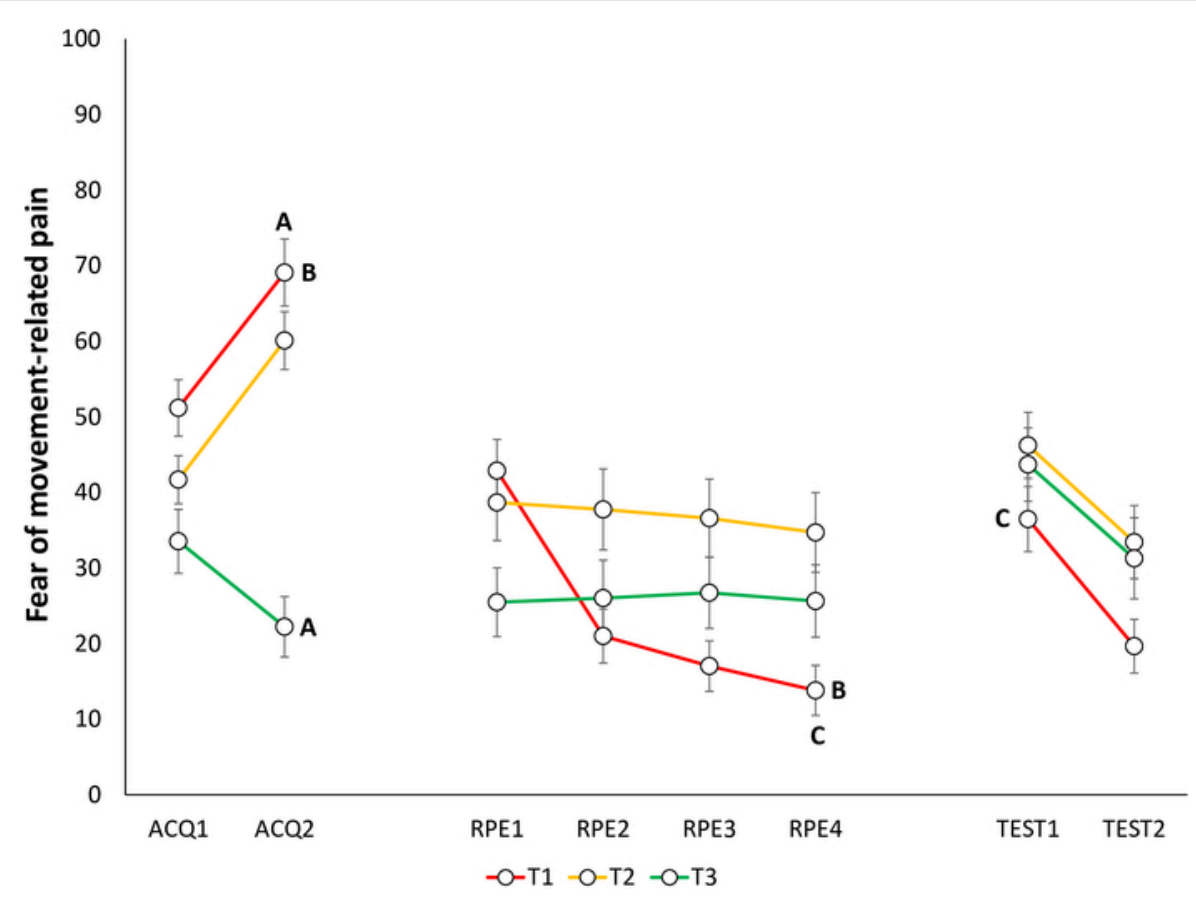

Figure 6: Representative data of fear of movement-related pain during the acquisition, extinction with response prevention, and test of spontaneous recovery phases ${ }^{22}$. Mean fear of movement-related pain toward the different trajectories (T1-T3) during acquisition (ACQ1-2), extinction with response prevention (RPE1-4), and spontaneous recovery (TEST1-2). Error bars represent SEM. Data in this figure are from 30 participants (9 men, 21 women; mean age $=21.90)^{22}$. This figure is modified with permission from ref. ${ }^{22}$. Please click here to view a larger version of this figure. 


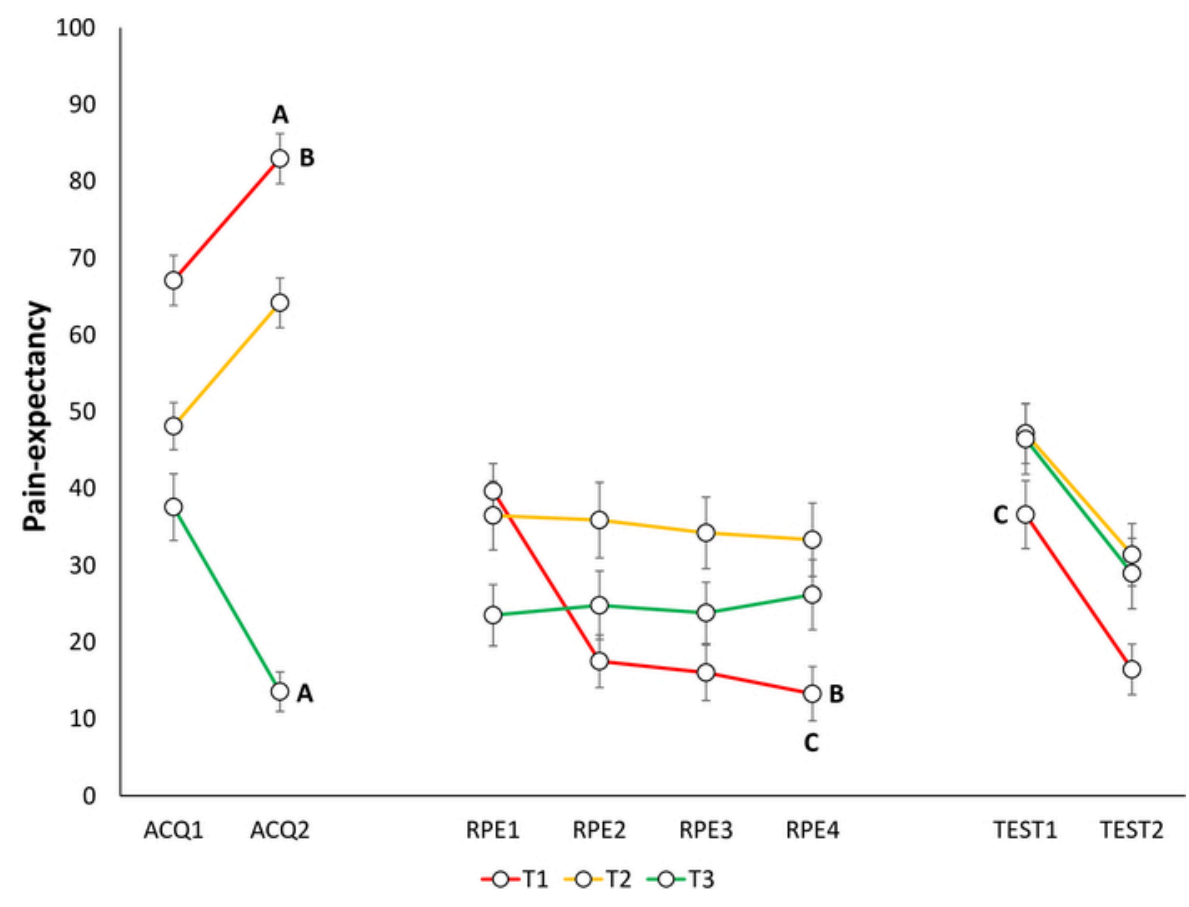

Figure 7: Representative data of pain-expectancy during the acquisition, extinction with response prevention, and test of spontaneous recovery phases ${ }^{22}$. Mean pain-expectancy toward the different trajectories (T1-T3) during acquisition (ACQ1-2), extinction with response prevention (RPE1-4), and spontaneous recovery (TEST1-2). Error bars represent SEM. Data in this figure are from 30 participants $(9 \text { men, } 21 \text { women; mean age }=21.90)^{22}$. This figure is modified with permission from ref. ${ }^{22}$. Please click here to view a larger version of this figure.

\section{Discussion}

Given the key role of avoidance in chronic pain disability $1,2,3,4,5$, and the limitations faced by traditional avoidance paradigms ${ }^{19}$, there is a need for methods to investigate (pain-related) avoidance behavior. The robotic arm-reaching paradigm presented here addresses a number of these limitations. We have employed the paradigm in a series of studies, which have consistently demonstrated acquisition of avoidance, and these effects have extended to our self-report measures of pain-expectancy and fear of movement-related pain $20,22,23,24$. However, we have also found dissociations between fear and avoidance ${ }^{23}$ that may be genuine and informative, suggesting that the two do not always share a one-to-one relationship $5,12,43,44,45$. Additionally, the paradigm presents multiple opportunities for investigating different aspects of avoidance behavior, such as generalization ${ }^{23}$, extinction with response prevention ${ }^{22}$, and post-extinction return of avoidance ${ }^{22}$, as described in the current manuscript.

The current method offers many advantages over traditional avoidance paradigms. First, instead of performing an experimenter-instructed avoidance response, participants in the robotic arm-reaching paradigm acquire avoidance behavior themselves. The paradigm thus better models real life situations, where avoidance behavior emerges naturally 
as a response to pain ${ }^{9}$. Understanding the processes underlying how avoidance is acquired, can provide insight into how avoidance can subsequently become pathological, and inspire ways in which these processes can be directly targeted during treatment ${ }^{51}$. For example, methodological modifications, such as manipulating experimental reward to increase approach and reduce avoidance tendencies ${ }^{52,53}$, can allow closer investigation of the behavioral and cognitive processes underlying the acquisition of maladaptive avoidance. With regard to this, the acquisition of avoidance demonstrated with the robotic arm-reaching paradigm can be easily applied to investigate excessive generalization of avoidance to safe stimuli ${ }^{23}$. A second advantage is that the continuous nature of the avoidance response in the current paradigm allows us to examine for whom avoidance might become excessive, as it provides more detailed data than a dichotomous measure. This increased detail in the data allows heightened sensitivity for picking up individual differences, by means of comparing deviation scores between participants. Such a continuous measure is also more ecologically valid, as avoidance in real life can occur at varying degrees. For example, pain-related avoidance can range from subtle (e.g., postural changes or changed breathing when performing a movement) to complete avoidance (e.g., being bedridden). Furthermore, in addition to incorporating a cost to avoidance, the current avoidance response demands some physical effort, meaning that costs increase with time throughout the task. This accurately models real life, where avoidance can become increasingly costly for the individual over a period of time ${ }^{9}$. For example, prolonged or regular absenteeism becomes costly from a financial point of view ${ }^{54,55}$. Finally, given the low cost associated with the previously used instructed button-press response, it is hard to disentangle whether participants in traditional avoidance paradigms avoid due to genuine fear, or simply due to automatic following of task instructions. In contrast, given the high-effort and uninstructed nature of the avoidance response in the current paradigm, it seems likely that any avoidance behavior observed models genuine self-motivated avoidance.

In addition to addressing limitations of previous methodologies, the robotic arm-reaching paradigm offers many opportunities for investigating further aspects of avoidance behavior, as demonstrated in the current manuscript by the avoidance generalization and RPE protocols. It is noteworthy that, we previously observed a dissociation between self-reports and avoidance, with fear and pain-expectancies generalizing to the novel movement trajectories, while avoidance did not. There are several plausible explanations for the observed discrepancy between fear and avoidance ${ }^{23}$, which we are currently investigating. However, this dissociation may also be a genuine and informative finding, which in fact adds to previous literature suggesting that fear and avoidance do not always occur in synchrony $5,12,43,44,45$, especially when the avoidance response is costly 56,57 . This finding emphasizes the importance of experimentally investigating avoidance behavior itself, as distinct processes most likely contribute to different aspects of fear learning 58,59 , and these processes would be difficult to uncover by solely measuring self-reports and psychophysiological indices of fear. In addition to generalization of avoidance to novel movements, the robotic arm-reaching paradigm has also been applied to study generalization of avoidance to novel contexts ${ }^{24}$. So far, context-based generalization of avoidance has been investigated using different colored screens as contextual cues ${ }^{24}$. However, Virtual Reality (VR) could be easily implemented with the current paradigm to increase the ecological validity of the experimental contexts. 
VR could also be applied to study category-based avoidance generalization, such as generalization of avoidance between different action categories ${ }^{60,61}$. Additional adaptations may also be implemented in the RPE protocol. Besides using a 2-day protocol for the investigation of spontaneous recovery $^{22}$, we have also investigated whether pain-related avoidance behavior returns not with the passage of time, but after unexpected encounters with the pain stimulus (reinstatement $^{42}$ in a 1-day protocol. Furthermore, to examine the proprioceptive underpinnings of pain-related avoidance behavior more closely, the paradigm can be modified to include less or no visual information. This is something we are currently investigating in our lab. Finally, given that physically moving away from an aversive stimulus represents a species-specific defensive response ${ }^{62}$, not unique to fear and pain, this type of operationalization of avoidance permits investigation of many different types of avoidance as well. For example, the paradigm can potentially be applied to examine, not only avoidance of painful stimuli, but also avoidance of other types of aversive stimuli, such as those inducing disgust or embarrassment ${ }^{63,64}$.

The described protocol can also be easily extended to include psychophysiological fear measures. Although not described here, we have incorporated eye-blink startle responses, as well as electroencephalography (EEG), into the robotic arm-reaching paradigm. The eye-blink startle measure offers a fear-specific measure of reflexive defensive responses ${ }^{65,66}$, which can provide additional insight into the mechanisms underlying avoidance behavior and its relationship to fear, whereas implementing EEG to the paradigm enables investigation into specific neural correlates of avoidance behavior ${ }^{67}$. Additionally, the skin-conductance response $(\mathrm{SCR})^{68}$, as well as online self-report ratings of relief-pleasantness ${ }^{69,70}$ could be included as measures of relief $^{71}$. SCRs have been previously found to correlate with relief $^{72}$-a proposed reinforcer of avoidance 69,70 given its inherent positive valence in response to the omission of negative events ${ }^{73}, 74$. Finally, heart rate $(H R)$ and heart rate variability (HRV) are easily implementable measures that have been linked to multiple aversive emotions associated with avoidance, such as fear, disgust, and embarrassment ${ }^{75}$.

Despite its strengths, we acknowledge that the robotic armreaching paradigm also has its limitations. For example, the paradigm is not easily transferable to other laboratories, as the equipment used in, and required for the paradigm (e.g., robot and constant current stimulator) are expensive, limiting the widespread use of the paradigm and its implementation by other laboratories. However, note that similar robots, which are relatively common in rehabilitation clinics, can be programmed in the same way, and more affordable constant current stimulators are available as well. It is also noteworthy that, in the current method the discriminative stimulus $\left(S^{D}\right)$ and the instrumental response are intertwined. This is in contrast to traditional avoidance paradigms, where fear is first acquired towards the CS during the Pavlovian phase, and avoidance is examined in a subsequent instrumental phase. However, the temporal relationship between fear and avoidance is not strictly unidirectional ${ }^{51}$. Although the current paradigm allows closer investigation of the temporal dynamics of avoidance-emergence in relation to fear-emergence, the measures we have employed so far do not allow us to accurately disentangle the temporal dynamics of fear and avoidance. Currently, avoidance behavior in the paradigm can be examined at a trialby-trial basis, whereas fear and expectancy ratings are only collected at discrete, specific time points during the task, to not interfere with task flow. However, to allow precise comparisons between fear and avoidance, a future 
study could use a more continuous measure of fear, for example, by means of a dial ${ }^{76}$, single-sensor $E E G^{77}$, or fear-potentiated startle, to allow a detailed understanding of fear-emergence towards the different trajectories, in relation to avoidance. Finally, only electrocutaneous stimuli have so far been used in the robotic arm-reaching paradigm as pain stimuli, for reasons of consistency and comparability with previous studies of pain-related fear $78,79,80$. However, electrocutaneous stimuli may not fully mimic the more tonic pain experienced by chronic pain patients, given that they produce a relatively phasic, uncommon, and unnatural pain experience 81 . Other pain-induction methods, such as ischemic stimulation ${ }^{82}$ and exercise-induced (e.g., delayed onset muscle soreness, DOMS) 83,84 pain have been argued to be better experimental analogues of musculoskeletal pain, given their natural and endogenous nature 81 . These paininduction methods could be employed in the robotic armreaching paradigm in the future. Despite these limitations, the ability of the current paradigm to consistently demonstrate acquisition of fear and avoidance using such entwined $S^{D} S$ and Rs is in itself interesting and novel. Furthermore, we believe that the robotic arm-reaching paradigm can in and of itself further the discussion of the need for more ecologically valid avoidance paradigms ${ }^{19}$. In addition, the paradigm has the potential to pave the way for developing better avoidance paradigms in general, by providing an example of how problems in the field can be tackled in an innovative manner.

In conclusion, the robotic arm-reaching paradigm offers a promising route to improving the ecological validity of investigations into avoidance behavior, and to furthering our understanding of the underlying processes. Using the paradigm, we have already obtained interesting results, which may not have been uncovered by solely assessing passive correlates of fear such as verbal reports and physiological arousal. Yet, extensions to the paradigm have provided some inconclusive results, which require further investigation and refinement of the procedure. Despite this, the robotic armreaching paradigm is a huge leap forward with respect to ecological validity in the paradigms used to study avoidance behavior.

\section{Disclosures}

The authors have nothing to disclose.

\section{Acknowledgments}

This research was supported by a Vidi grant from the Netherlands Organization for Scientific Research (NWO), The Netherlands (grant ID 452-17-002) and a Senior Research Fellowship of the Research Foundation Flanders (FWOVlaanderen), Belgium (grant ID: 12E3717N) granted to Ann Meulders. The contribution of Johan Vlaeyen was supported by the "Asthenes" long-term structural funding Methusalem grant by the Flemish Government, Belgium.

The authors wish to thank Jacco Ronner and Richard Benning from Maastricht University, for programming the experimental tasks, and designing and creating the graphics for the described experiments.

\section{References}

1. Crombez, G., Eccleston, C., Van Damme, S., Vlaeyen, J. W., Karoly, P. Fear-avoidance model of chronic pain: the next generation. The Clinical Journal of Pain. 28 (6), 475-483 (2012).

2. Leeuw, M. et al. The fear-avoidance model of musculoskeletal pain: current state of scientific evidence. Journal of Behavioral Medicine. 30 (1), 77-94 (2007). 
3. Vlaeyen, J., Linton, S. Fear-avoidance model of chronic musculoskeletal pain: 12 years on. Pain. 153 (6), 1144-1147 (2012).

4. Vlaeyen, J., Linton, S. Fear-avoidance and its consequences in chronic musculoskeletal pain: a state of the art. Pain. 85 (3), 317-332 (2000).

5. Meulders, A. From fear of movement-related pain and avoidance to chronic pain disability: a state-of-the-art review. Current Opinion in Behavioral Sciences. 26, 130-136 (2019).

6. Kori, S. H., Miller, R. P., Todd, D. D. Kinesophobia: a new view of chronic pain behavior. Pain Management. (3), 35-43 (1990).

7. Lethem, J., Slade, P. D., Troup, J. D., Bentley, G. Outline of a Fear-Avoidance Model of exaggerated pain perception-I. Behaviour Research and Therapy. 21 (4), 401-408 (1983).

8. Waddell, G., Newton, M., Henderson, I., Somerville, D., Main, C. J. A Fear-Avoidance Beliefs Questionnaire $(F A B Q)$ and the role of fear-avoidance beliefs in chronic low back pain and disability. Pain. 52 (2), 157-168 (1993).

9. Volders, S., Boddez, Y., De Peuter, S., Meulders, A., Vlaeyen, J. W. Avoidance behavior in chronic pain research: a cold case revisited. Behaviour Research and Therapy. 64, 31-37 (2015).

10. Lovibond, P. F., Mitchell, C. J., Minard, E., Brady, A., Menzies, R. G. Safety behaviours preserve threat beliefs: Protection from extinction of human fear conditioning by an avoidance response. Behaviour Research and Therapy. 47 (8), 716-720 (2009).
11. Hofmann, S. G., Hay, A. C. Rethinking avoidance: Toward a balanced approach to avoidance in treating anxiety disorders. Journal of Anxiety Disorders. 55, 14-21 (2018).

12. Krypotos, A. M., Effting, M., Kindt, M., Beckers, T. Avoidance learning: a review of theoretical models and recent developments. Frontiers in Behavioral Neuroscience. 9, 189 (2015).

13. Mowrer, O. H. Two-factor learning theory: summary and comment. Psychological Review. 58 (5), 350-354 (1951).

14. Pavlov, I. P. Conditioned reflexes: An investigation of the physiological activity of the cerebral cortex. Oxford University Press. (1927).

15. Skinner, B. F. Science and human behavior. Macmillan. (1953).

16. Thorndike, E. L. Animal intelligence: An experimental study of the associative processes in animals. The Psychological Review: Monograph Supplements. 2 (4), i-109 (1898).

17. Linton, S. J., Götestam, K. G. Controlling pain reports through operant conditioning: a laboratory demonstration. Perceptual and Motor Skills. 60 (2), 427-437 (1985).

18. Gatzounis, R., Schrooten, M. G., Crombez, G., Vlaeyen, J. W. Operant learning theory in pain and chronic pain rehabilitation. Current Pain and Headache Reports. 16 (2), 117-126 (2012).

19. Krypotos, A. M., Vervliet, B., Engelhard, I. M. The validity of human avoidance paradigms. Behaviour Research and Therapy. 111 99-105 (2018).

20. Meulders, A., Franssen, M., Fonteyne, R., Vlaeyen, J. Acquisition and extinction of operant pain-related avoidance behavior using a 3 degrees-of-freedom robotic arm. Pain. 157 (5) (2016). 
21. Boddez, Y. et al. Rating data are underrated: Validity of US expectancy in human fear conditioning. Journal of Behavior Therapy and Experimental Psychiatry. 44 (2), 201-206 (2013).

22. Gatzounis, R., Meulders, A. Once an Avoider Always an Avoider? Return of Pain-Related Avoidance After Extinction With Response Prevention. The Journal of Pain. (2020).

23. Glogan, E., Gatzounis, R., Meulders, M., Meulders, A. Generalization of instrumentally acquired pain-related avoidance to novel but similar movements using a robotic arm-reaching paradigm. Behaviour Research and Therapy. 124, 103525 (2020).

24. Meulders, A., Franssen, M., Claes, J. Avoiding Based on Shades of Gray: Generalization of Pain-Related Avoidance Behavior to Novel Contexts. The Journal of Pain. (2020).

25. Kalish, H. I. in Learning: processes. ed M. Marx. 207-297. Macmillan (1969).

26. Honig, W. K., Urcuioli, P. J. The legacy of Guttman and Kalish (1956): Twenty-five years of research on stimulus generalization. Journal of the Experimental Analysis of Behavior. 36 (3), 405-445 (1981).

27. Ghirlanda, S., Enquist, M. A century of generalization. Animal Behaviour. 66 (1), 15-36 (2003).

28. Dymond, S., Dunsmoor, J., Vervliet, B., Roche, B., Hermans, D. Fear generalization in humans: Systematic review and implications for anxiety disorder research. Behavior Therapy. 46 (5), 561-582 (2015).

29. Lissek, S., Grillon, C. Overgeneralization of conditioned fear in the anxiety disorders. Zeitschrift für Psychologie/ Journal of Psychology. 218 (2), 146-148 (2010).
30. Meulders, A. et al. Contingency learning deficits and generalization in chronic unilateral hand pain patients. The Journal of Pain. 15 (10), 1046-1056 (2014).

31. Meulders, A., Jans, A., Vlaeyen, J. Differences in pain-related fear acquisition and generalization: an experimental study comparing patients with fibromyalgia and healthy controls. Pain. 156 (1), 108-122 (2015).

32. Meulders, A., Meulders, M., Stouten, I., De Bie, J., Vlaeyen, J. W. Extinction of fear generalization: A comparison between fibromyalgia patients and healthy control participants. The Journal of Pain. 18 (1), 79-95 (2017).

33. Harvie, D. S., Moseley, G. L., Hillier, S. L., Meulders, A. Classical Conditioning Differences Associated With Chronic Pain: A Systematic Review. The Journal of Pain. 18 (8), 889-898 (2017).

34. Meulders, A. Fear in the context of pain: Lessons learned from 100 years of fear conditioning research. Behaviour Research and Therapy. 131, 103635 (2020).

35. Vlaeyen, J., Morley, S., Linton, S., Boersma, K., de Jong, J. Pain-Related Fear: Exposure Based Treatment for Chronic Pain. IASP Press, (2012).

36. Scheveneels, S., Boddez, Y., Vervliet, B., Hermans, D. The validity of laboratory-based treatment research: Bridging the gap between fear extinction and exposure treatment. Behaviour Research and Therapy. 86, 87-94 (2016).

37. den Hollander, M. et al. Fear reduction in patients with chronic pain: a learning theory perspective. Expert Review of Neurotherapeutics. 10 (11), 1733-1745 (2010). 
38. Craske, M. G. et al. Optimizing inhibitory learning during exposure therapy. Behaviour Research Therapy. 46 (1), 5-27 (2008).

39. Quirk, G. J., Mueller, D. Neural mechanisms of extinction learning and retrieval. Neuropsychopharmacology: An Official Publication of the American College of Neuropsychopharmacology. 33 (1), 56-72 (2008).

40. Bouton, M., E. Context, ambiguity, and unlearning: sources of relapse after behavioral extinction. Biological Psychiatry. 52 (10), 976-986 (2002).

41. Bouton, M. E., Winterbauer, N. E., Todd, T. P. Relapse processes after the extinction of instrumental learning: renewal, resurgence, and reacquisition. Behavioural processes. 90 (1), 130-141 (2012).

42. Haaker, J., Golkar, A., Hermans, D., Lonsdorf, T. B. A review on human reinstatement studies: an overview and methodological challenges. Learning \& Memory. 21 (9), 424-440 (2014).

43. Mineka, S. The role of fear in theories of avoidance learning, flooding, and extinction. Psychological Bulletin. 86 (5), 985-1010 (1979).

44. Bravo-Rivera, C., Roman-Ortiz, C., MontesinosCartagena, M., Quirk, G. J. Persistent active avoidance correlates with activity in prelimbic cortex and ventral striatum. Frontiers In Behavioral Neuroscience. 9, 184-184 (2015).

45. Vervliet, B., Indekeu, E. Low-cost avoidance behaviors are resistant to fear extinction in humans. Frontiers In Behavioral Neuroscience. 9, 351 (2015).

46. Solomon, R. L., Kamin, L. J., Wynne, L. C. Traumatic avoidance learning: the outcomes of several extinction procedures with dogs. The Journal of Abnormal and Social Psychology. 48 (2), 291-302 (1953).

47. Bouton, M., E., Swartzentruber, D. Sources of relapse after extinction in Pavlovian and instrumental learning. Clinical Psychology Review. 11 (2), 123-140 (1991).

48. Davis, J., Bitterman, M. E. Differential reinforcement of other behavior (DRO): a yoked-control comparison. Journal of the Experimental Analysis of Behavior. 15 (2), 237-241 (1971).

49. Bouton, M., E., Todd, T., P. A fundamental role for context in instrumental learning and extinction. Behavioural Processes. 104, 13-19 (2014).

50. Bouton, M., E., Todd, T., P., Leon, S., P. Contextual control of discriminated operant behavior. The Journal of Experimental Psychology: Animal Learning and Cognition. 40 (1), 92-105 (2014).

51. Pittig, A., Wong, A. H. K., Glück, V. M., Boschet, J. M. Avoidance and its bi-directional relationship with conditioned fear: Mechanisms, moderators, and clinical implications. Behaviour Research and Therapy. 126, 103550, (2020).

52. Pittig, A., Dehler, J. Same fear responses, less avoidance: Rewards competing with aversive outcomes do not buffer fear acquisition, but attenuate avoidance to accelerate subsequent fear extinction. Behaviour Research and Therapy. 112, 1-11 (2019).

53. Van Damme, S., Van Ryckeghem, D. M., Wyffels, F., Van Hulle, L., Crombez, G. No pain no gain? Pursuing a competing goal inhibits avoidance behavior. Pain. 153 (4), 800-804 (2012).

54. Langley, P. et al. The impact of pain on labor force participation, absenteeism and presenteeism in the 
European Union. Journal of Medical Economics. 13 (4), 662-672 (2010).

55. Breivik, H., Collett, B., Ventafridda, V., Cohen, R., Gallacher, D. Survey of chronic pain in Europe: prevalence, impact on daily life, and treatment. European Journal of Pain. 10 (4), 287-333 (2006).

56. Claes, N., Crombez, G., Vlaeyen, J. W. Pain-avoidance versus reward-seeking: an experimental investigation. Pain. 156 (8), 1449-1457 (2015).

57. Claes, N., Karos, K., Meulders, A., Crombez, G., Vlaeyen, J.W. S. Competing goals attenuate avoidance behavior in the context of pain. The Journal of Pain. 15 (11), 1120-1129 (2014).

58. Soeter, M., Kindt, M. Dissociating response systems: erasing fear from memory. Neurobiology of Learning and Memory. 94 (1), 30-41 (2010).

59. LeDoux, J., Daw, N. D. Surviving threats: neural circuit and computational implications of a new taxonomy of defensive behaviour. Nature Reviews Neuroscience. 19 (5), 269-282 (2018).

60. Glogan, E., van Vliet, C., Roelandt, R., Meulders, A. Generalization and extinction of concept-based painrelated fear. The Journal of Pain. 20 (3), 325-338 (2019).

61. Meulders, A., Vandael, K., Vlaeyen, J. W. Generalization of Pain-Related Fear Based on Conceptual Knowledge. Behavior Therapy. 48 (3), 295-310 (2017).

62. Bolles, R. C. Species-specific defense reactions and avoidance learning. Psychological Review. 77 (1), 32-48 (1970).

63. Shook, N. J., Thomas, R., Ford, C. G. Testing the relation between disgust and general avoidance behavior.
Personality and Individual Differences. 150, 109457 (2019).

64. McCambridge, S. A., Consedine, N. S. For whom the bell tolls: Experimentally-manipulated disgust and embarrassment may cause anticipated sexual healthcare avoidance among some people. Emotion. 14 (2), 407-415 (2014).

65. Lipp, O. V., Sheridan, J., Siddle, D. A. Human blink startle during aversive and nonaversive Pavlovian conditioning. The Journal of Experimental Psychology: Animal Learning and Cognition. 20 (4), 380-389 (1994).

66. van Well, S., Visser, R. M., Scholte, H. S., Kindt, M. Neural substrates of individual differences in human fear learning: evidence from concurrent fMRI, fear-potentiated startle, and US-expectancy data. Cognitive, Affective, \& Behavioral Neuroscience. 12 (3), 499-512 (2012).

67. Davidson, R. J., Jackson, D. C., Larson, C. L. in Handbook of psychophysiology, 2nd ed. Cambridge University Press. 27-52 (2000).

68. Benedek, M., Kaernbach, C. A continuous measure of phasic electrodermal activity. Journal of Neuroscience Methods. 190 (1), 80-91 (2010).

69. Leknes, S., Lee, M., Berna, C., Andersson, J., Tracey, I. Relief as a reward: hedonic and neural responses to safety from pain. PloS One. 6 (4), e17870-e17870 (2011).

70. Vervliet, B., Lange, I., Milad, M. R. Temporal dynamics of relief in avoidance conditioning and fear extinction: Experimental validation and clinical relevance. Behaviour Research and Therapy. 96, 66-78 (2017).

71. Leknes, S. et al. The importance of context: When relative relief renders pain pleasant. PAIN. 154 (3), 402-410 (2013). 
72. Vervliet, B., Lange, I., Milad, M. R. Temporal dynamics of relief in avoidance conditioning and fear extinction: Experimental validation and clinical relevance. Behaviour Research and Therapy. 96, 66-78 (2017).

73. Deutsch, R., Smith, K. J. M., Kordts-Freudinger, R., Reichardt, R. How absent negativity relates to affect and motivation: an integrative relief model. Frontiers in Psychology. 6 (152), (2015).

74. Vlemincx, E. et al. Why do you sigh? Sigh rate during induced stress and relief. Psychophysiology. 46 (5), 1005-1013 (2009).

75. Kreibig, S. D. Autonomic nervous system activity in emotion: A review. Biological Psychology. 84 (3), 394-421 (2010).

76. Pappens, M., Smets, E., Vansteenwegen, D., Van Den Bergh, O., Van Diest, I. Learning to fear suffocation: a new paradigm for interoceptive fear conditioning. Psychophysiology. 49 (6), 821-828 (2012).

77. de Man, J., Stassen, N. Analyzing fear using single sensor EEG device. in International Conference on Intelligent Technologies for Interactive Entertainment. eds R Poppe, Meyer J.J., Veltkamp R., Dastani M. 86-96 Springer. (2016).

78. Meulders, A., Vandebroek, N., Vervliet, B., Vlaeyen, J.W.S. Generalization Gradients in Cued and Contextual Pain-Related Fear: An Experimental Study in Healthy Participants. Frontiers in Human Neuroscience. 7, 345 (2013).

79. Meulders, A., Vansteenwegen, D., Vlaeyen J. W. S. The acquisition of fear of movement-related pain and associative learning: a novel pain-relevant human fear conditioning paradigm. Pain. 152 (11), 2460-2469 (2011).
80. Meulders, A., Vlaeyen, J. W. S. The acquisition and generalization of cued and contextual pain-related fear: an experimental study using a voluntary movement paradigm. Pain. 154 (2), 272-282 (2013).

81. Moore, D. J., Keogh, E., Crombez, G., Eccleston, C. Methods for studying naturally occurring human pain and their analogues. Pain. 154 (2), 190-199 (2013).

82. Lewis, T. Pain in muscular ischemia: its relation to anginal pain. Archives of Internal Medicine. 49 (5), 713-727 (1932).

83. Niederstrasser, N. G. et al. Pain catastrophizing and fear of pain predict the experience of pain in body parts not targeted by a delayed-onset muscle soreness procedure. The Journal of Pain. 16 (11), 1065-1076 (2015).

84. Niederstrasser, N. G. et al. An experimental approach to examining psychological contributions to multisite musculoskeletal pain. The Journal of Pain. 15 (11), 1156-1165 (2014). 